\title{
Clinical Influence of Cervical Spinal Canal Stenosis on Neurological Outcome after Traumatic Cervical Spinal Cord Injury without Major Fracture or Dislocation
} \author{
Eiji Mori ${ }^{1}$, Itaru Yugue ${ }^{1}$, Osamu Kawano ${ }^{1}$, Hiroaki Sakai ${ }^{1}$, Takayoshi Ueta ${ }^{1}$, Keiichiro Shiba ${ }^{1}$ \\ ${ }^{I}$ Department of Orthopaedic Surgery, Spinal Injuries Center, Iizuka, Japan \\ ${ }^{2}$ Department of Orthopaedic Surgery, Kyushu University, Fukuoka, Japan \\ ${ }^{3}$ Department of Rehabilitation Medicine, Spinal Injuries Center, Iizuka, Japan
}

Tsuneaki Takao ${ }^{1}$, Seiji Okada ${ }^{2}$, Yuichiro Morishita ${ }^{1}$, Takeshi Maeda ${ }^{1}$, Kensuke Kubota $^{2}$, Ryosuke Ideta ${ }^{3}$,

Study Design: Retrospective case series.

Purpose: To clarify the influence of cervical spinal canal stenosis (CSCS) on neurological functional recovery after traumatic cervical spinal cord injury (CSCI) without major fracture or dislocation

Overview of Literature: The biomechanical etiology of traumatic CSCI remains under discussion and its relationship with CSCS is one of the most controversial issues in the clinical management of traumatic CSCl.

Methods: To obtain a relatively uniform background, patients non-surgically treated for an acute C3-4 level CSCI without major fracture or dislocation were selected. We analyzed 58 subjects with traumatic CSCI using T2-weighted mid-sagittal magnetic resonance imaging. The sagittal diameter of the cerebrospinal fluid (CSF) column, degree of canal stenosis, and neurologic outcomes in motor function, including improvement rate, were assessed.

Results: There were no significant relationships between sagittal diameter of the CSF column at the C3-4 segment and their American Spinal Injury Association motor scores at both admission and discharge. Moreover, no significant relationships were observed between the sagittal diameter of the CSF column at the C3-4 segment and their neurological recovery during the following period. Conclusions: No relationships between pre-existing CSCS and neurological outcomes were evident after traumatic CSCI. These results suggest that decompression surgery might not be recommended for traumatic CSCl without major fracture or dislocation despite pre-existing CSCS.

Keywords: Cervical spinal canal stenosis; Cervical spinal cord injury without major fracture or dislocation; Magnetic resonance imaging; Neurological outcome

\section{Introduction}

Numerous previous studies have been published concern- ing adult traumatic cervical spinal cord injuries (CSCI) without major bony injury or dislocation, using various nomenclature, such as hyperextension dislocation [1],

Received Sep 13, 2015; Revised Oct 25, 2015; Accepted Oct 26, 2015

Corresponding author: Tsuneaki Takao

Department of Orthopaedic Surgery, Spinal Injuries Center, 550-4 Igisu, Iizuka 820-8508, Japan

Tel: +81-948-24-7500, Fax: +81-948-29-1065, E-mail: tsuneaki@iris.ocn.ne.jp 
Table 1. Neurological status (ASIA impairment score) at admission and discharge

\begin{tabular}{|c|c|c|c|c|c|c|}
\hline \multirow{2}{*}{ On admission } & \multirow{2}{*}{ No. } & \multicolumn{5}{|c|}{ On discharge } \\
\hline & & A & $\mathrm{B}$ & $C$ & $\mathrm{D}$ & $E$ \\
\hline A & 10 & 2 & 1 & 6 & 1 & - \\
\hline B & 11 & - & 1 & 6 & 4 & - \\
\hline C & 31 & - & - & 1 & 30 & - \\
\hline D & 6 & - & - & - & 5 & 1 \\
\hline
\end{tabular}

ASIA, American Spinal Injuries Association.

spinal cord injury (SCI) without radiographical abnormality in adults (SCIWORA) [2-6], SCI without radiographical evidence of trauma (SCIWORET) [7-9], and CSCI without bony injury [10-11]. Most patients are elderly and may present with tetraplegia caused by a hyperextension injury, predominantly at the C3-4 segment, with cord compression as a result of a stenotic spondylotic canal [3,7,12-14]. However, the influence of cervical spinal canal stenosis (CSCS) on neurological recovery after CSCI remains unclear.

The broad definition of SCIWORA/SCIWORET includes disc injury, anterior vertebral body tip or spinous process fracture, or other ligamentous injury. We defined CSCI with or without those injuries but without spinal canal bony injury as traumatic CSCI without major fracture or dislocation. Traumatic CSCI can occur with or without CSCS and cervical cord compression. The biomechanical etiology of traumatic CSCI without major fracture or dislocation remains under discussion, and its relationship with CSCS is one of the most controversial issues in the clinical management of traumatic CSCI.

The aims of the current study were to clarify the influence of spinal canal stenosis on neurological functional recovery after traumatic CSCI without major fracture or dislocation.

\section{Materials and Methods}

\section{Study population}

A total of 101 subjects with traumatic CSCI without major fracture or dislocation were treated conservatively in our facility from 2010 to 2013. All patients underwent functional plain radiographs including flexion and extension, computed tomography (CT), magnetic resonance imaging (MRI), and neurologic examination by a senior spine surgeon at the time of admission. All patients wore a Philadelphia collar for 4 weeks and started their rehabilitation from sitting exercise immediately without causing any discomfort if their general conditions were stable. Of these patients, 58 had the injury at C3-4 (57.4\%); 1 at C2-3 (1.0\%); 26 at C4-5 (25.7\%); 12 at C5-6 (11.9\%); and 4 at C6-7 (4.0\%). In the study, we focused on the subjects with responsible injured segment at the C3-4 segment who were admitted to our facility within 48 hours following trauma and had evidence of cervical cord injury with cervical cord intensity change on T2-weighted MRI at the C3-4 segment. A total of 58 subjects (52 men, 6 women; average age, 63.8 years [range, $42-89$ years]) were included in the study. Of the 58 patients, 34 showed central cord syndrome, 20 showed transverse, 2 showed anterior cord syndrome, and 2 showed Brown-Sequard syndrome. The average period of hospitalization was 258 days (range, 61 to 550). The neurologic status of the patients at the time of admission and discharge are summarized in Table 1. Patients with the following conditions were excluded from the study: multiple segmental cervical cord injury, cervical myelopathy before trauma, apparent herniated disc at the injured segment, severe instability on functional radiographs as defined by White et al. [15] (dynamic translation $>3.5 \mathrm{~mm}$ or $11^{\circ}$ greater angulation than that in the adjacent segment) or indication of spontaneous reduction of dislocation at C3-4 segment, or ankylosing spondylitis.

Institutional Review Board approval was granted and informed consent was obtained from all patients included in the study.

\section{Measurement of sagittal diameter of the CSF column}

We used a T2-weighted mid-sagittal MRI scan obtained at the time of admission to measure the sagittal diameters of the cerebrospinal fluid (CSF) column at the C3-4 
intervertebral disc level and C3 pedicle level. The rate of spinal canal stenosis was calculated using the following equation: (A-B)/A×100 (Fig. 1).

\section{Neurological status (ASIA motor score)}

The American Spinal Injuries Association (ASIA) motor

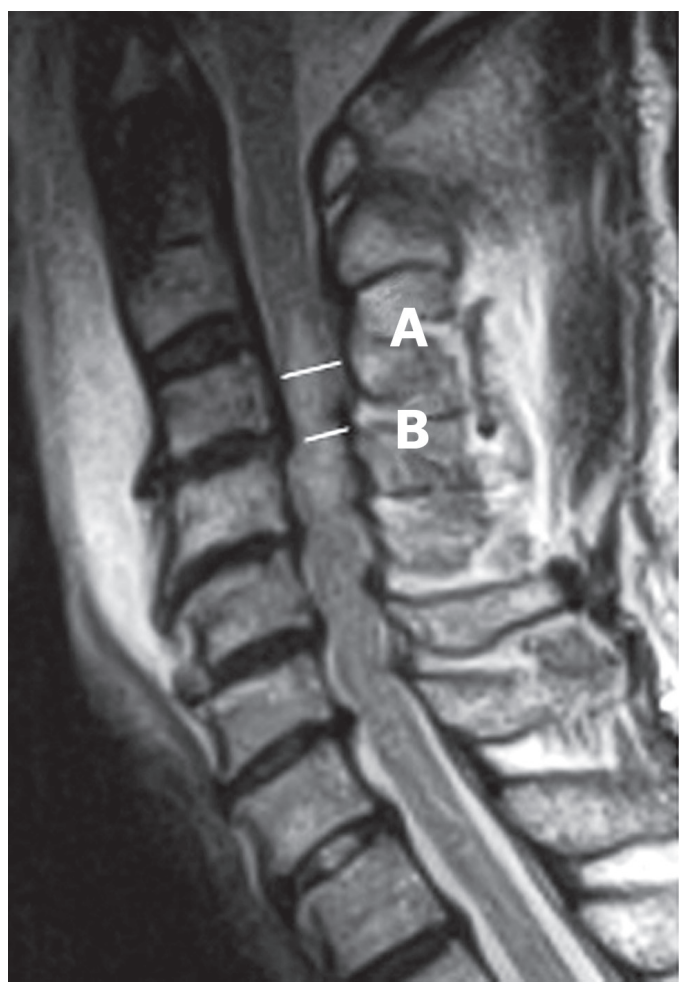

Fig. 1. T2-weighted midsagittal magnetic resonance imaging. $A$ is the diameter of the cervical cord at the noncompression level and $B$ is the diameter of the cervical cord at the injured level.

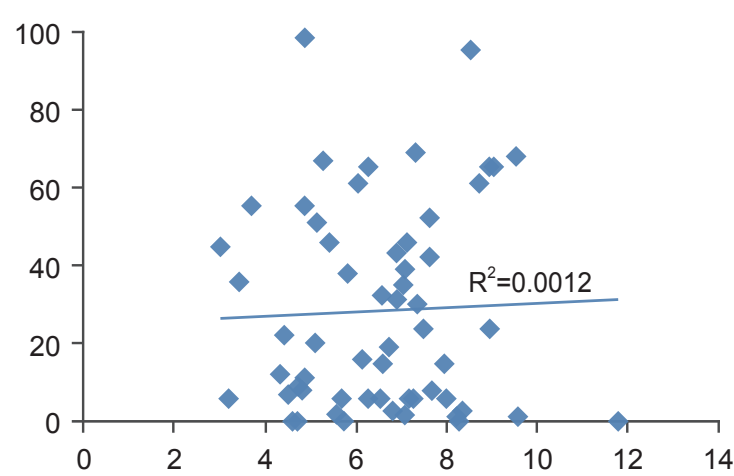

A Diameter of the cervical CSF column ( $\mathrm{mm})$ score (range, 0 to 100) was documented at the time of admission and discharge for each patient. Neurological recovery was evaluated as an improvement rate (\%) calculated as (motor score at discharge-motor score at admission $) /(100$-motor score at admission $) \times 100$.

\section{Statistical analysis}

Statistical analysis was performed using the Spearman rank-correlation coefficient to evaluate the relationships between neurological status and CSCS. $p<0.05$ was considered statistically significant.

\section{Results}

The average value of the sagittal diameters of the CSF columns at the C3-4 intervertebral disc and C3 pedicle level was $6.5 \mathrm{~mm}$ and $8.9 \mathrm{~mm}$, respectively. The average period of hospitalization was 258 days (range, 61 to 550 days). The average value of ASIA motor score at the time of admission and discharge was 28.5 and 67.7, respectively, and the improvement rate was $61.1 \%$. The average value of the spinal canal stenosis ratio was $25.7 \%$.

Fig. 2 shows the relationships between the sagittal diameter of the cervical CSF column at the C3-4 intervertebral disc level and ASIA motor score, which reflects neurological status at the time of admission and discharge. There were no significant relationships between the sagittal diameter of the cervical CSF column and ASIA motor scores both at the time of admission $(p=0.773)$ and discharge ( $p=0.138$ ) for the subjects with traumatic CSCI.

Fig. 3 shows the relationship between the sagittal diam-

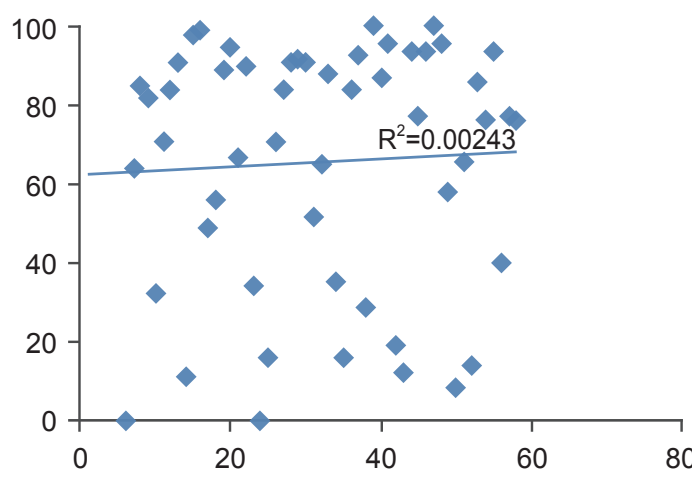

B Diameter of the cervical CSF column (mm)

Fig. 2. The sagittal diameter of the cervical cerebrospinal fluid (CSF) column and American Spinal Injuries Association motor score (A) at admission and (B) at discharge. 


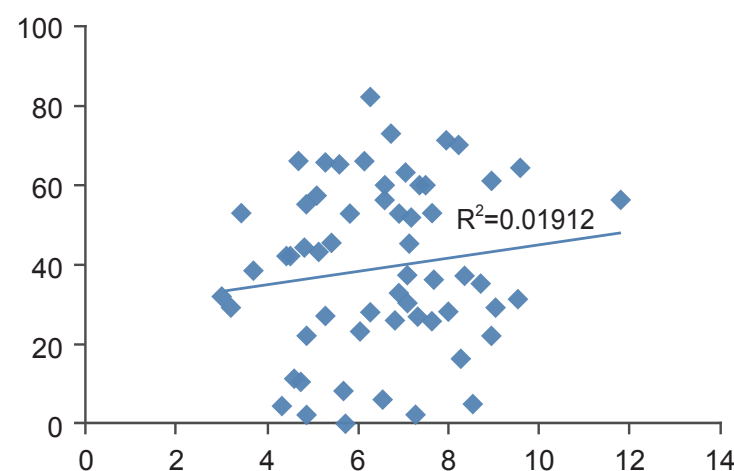

A Diameter of the cervical CSF column ( $\mathrm{mm}$ )

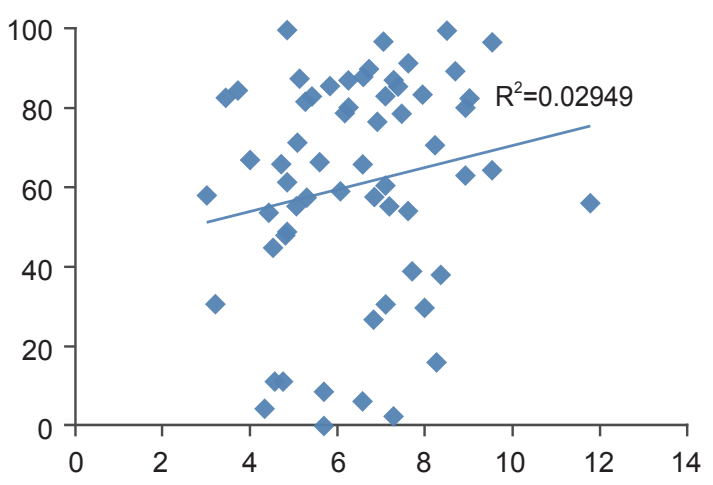

(B) Diameter of the cervical CSF column ( $\mathrm{mm})$

Fig. 3. Sagittal diameter of the cervical cerebrospinal fluid (CSF) column and the improvement rate according to the (A) subtraction score and (B) improvement rate (\%).

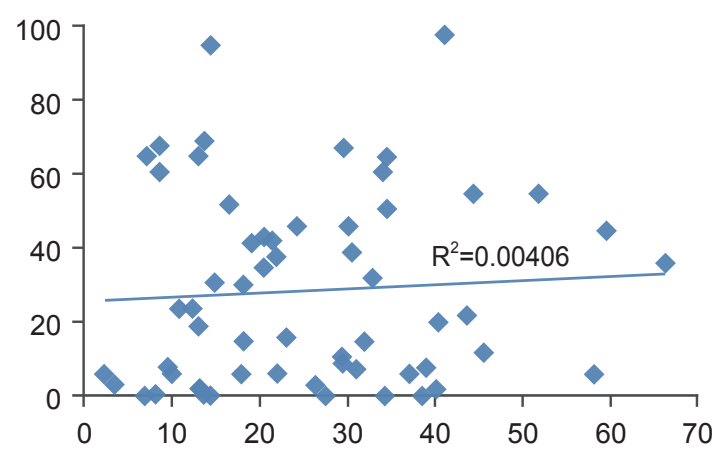

(A) Rate of spinal cord stenosis (\%)

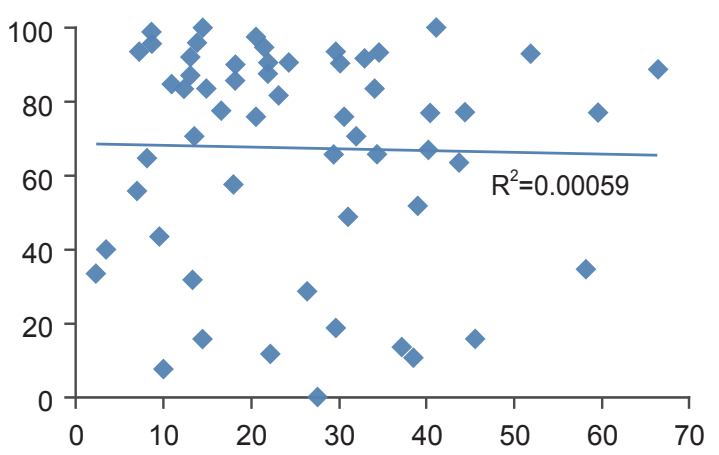

(B) Rate of spinal cord stenosis (\%)

Fig. 4. The rate of spinal cord stenosis and American Spinal Injuries Association motor score (A) at admission and (B) at discharge.

eter of the cervical CSF column at the C3-4 intervertebral disc level and the improvement rate, which reflects neurological recovery at the time of discharge. We defined the subtraction score as (motor score on discharge)-(motor score on admission). There was no significant relationship between the sagittal diameter of the cervical CSF column and neurological recovery at the time of discharge (subtraction score; $p=0.155$, improvement rate; $p=0.111$ ).

Fig. 4 shows the relationships between the rate of spinal canal stenosis and ASIA motor scores. There were no significant relationships between the rate of spinal canal stenosis and ASIA motor scores both at the time of admission $(p=0.897)$ and discharge $(p=0.315)$.

Fig. 5 shows the relationship between the rate of spinal canal stenosis and improvement rate. There was also no significant relationship between the rate of spinal canal stenosis and neurological recovery at the time of discharge (subtraction score; $p=0.441$, improvement rate; $p=0.277$ ).

\section{Discussion}

The number of CSCI without major fracture or dislocation has been increasing as the population ages [16]. However, the clinical management of traumatic CSCI is contentious.

Some authors recommend surgery for patients with pre-existing canal stenosis, as persistent cord compression may hinder neurological improvement [17-19]. La Rosa et al. [17] reported that early decompression surgery within 24 hours after trauma had a significantly better outcome compared with late surgical management. Chen et al. [18] recommended surgical treatment to achieve rapid neurological recovery and shorter hospitalization despite the level of functional recovery in the surgical and conserva- 

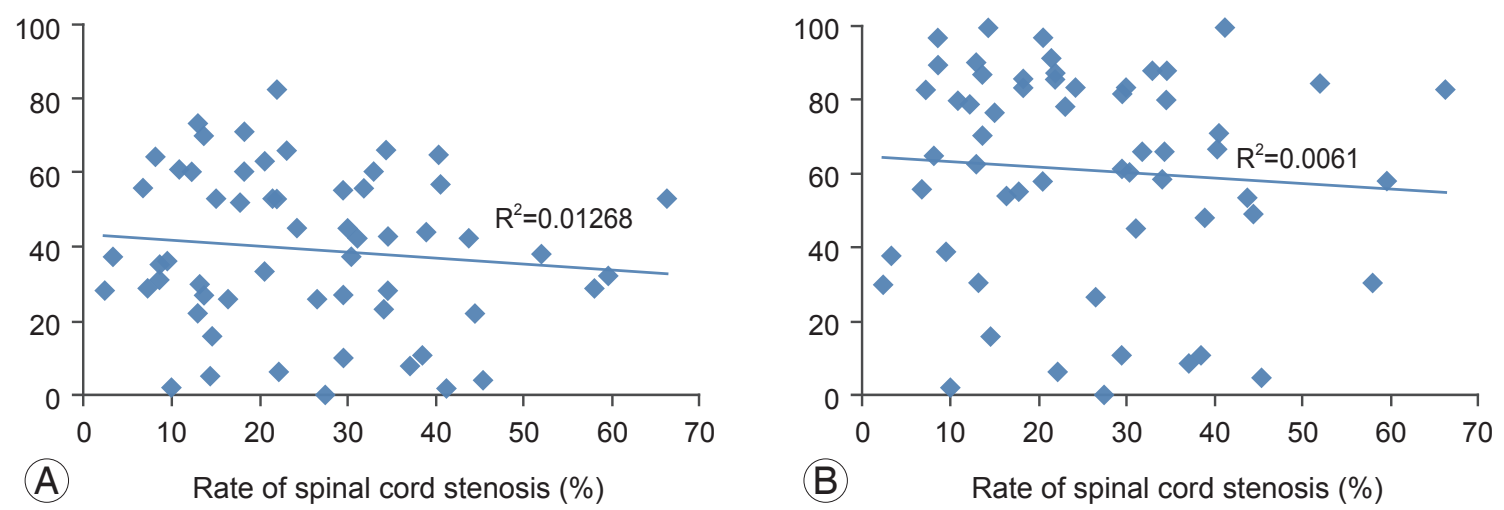

Fig. 5. The rate of spinal cord stenosis and the improvement rate according to the (A) subtraction score and (B) improvement rate $(\%)$.

tive treatment groups. Yamazaki et al. [19] reported that the canal diameter was a reliable predictor of recovery and recommended early decompression surgery. However, these studies were retrospective comparisons with small data sets and so likely had an inherent selection bias for surgical management.

In contrast, other researchers have reported no additional benefit with surgery compared to conservative treatment [20-22]. For example, Itoh et al. [20] reported no significant difference in neurological improvement between surgical and conservative management of CSCI without major bony injury; a higher frequency of postoperative complications was observed in subjects who were treated surgically. Kawano et al. [21] reported that surgical treatment was not superior to conservative treatment for CSCI without major bony injury with spinal cord compression in the acute phase. Although this study is not a comparison of conservative and operative treatment, our results propound prudence for surgical decompression until unequivocal evidence demonstrating improved neurological recovery or the prevention of delayed deterioration through surgery is presented.

In our facility, CSCI patients without major fracture or dislocation have consistently been treated conservatively and rehabilitated as early as possible, thus providing an appropriate study cohort to examine whether cervical canal stenosis is a risk factor for a deteriorative neurological course. We focused on the subjects with CSCI injured at C3-4 segment because of its high frequency (57.4\%). Several studies have reported the frequent incidence of traumatic CSCI at the level of C3-4 segment. However, under the circumstances, this remains a matter of debate. In the study, there were no significant relationships between sagittal C3-4 cord diameters and ASIA motor scores both at the time of admission and discharge. Moreover, no significant relationship between CSCS and neurological recovery was seen in CSCI subjects. These results appear to be unreasonable, since pathophysiology is closely related with stenotic factors, especially in degenerative cervical myelopathy $[23,24]$. Yet, careful consideration of the mechanism of traumatic SCI could provide accountable interpretations. At the moment of traumatic injury, the unphysiological and instantaneous dynamic stenosis would most significantly affect the neurological outcomes [16]. In the present study, what we consider to be most important finding is that canal stenosis on radiographs is a different condition from the unphysiological stenosis at the moment of injury. For this reason, the neurological outcome varies greatly, even among patients with the same canal diameter. Our data are consistent with those of earlier studies that showed that motor deficit severity does not statistically correlate with spinal canal stenosis degree $[16,25,26]$. Our results suggest that decompression surgery might not be necessary for CSCI without major fracture or dislocation with asymptomatic pre-existing stenosis in the acute phase.

Nevertheless, some questions remain unanswered even after the current study and so this study can only serve as a pilot study for further research using larger populations, which may help resolve several issues not addressed in this study and further clarify the clinical management of traumatic CSCI without major fracture or dislocation.

\section{Conclusions}

Our results showed no relationships between pre-existing 
CSCS and neurological outcomes after traumatic CSCI. These results suggested that decompression surgery might not be recommended for traumatic CSCI without major fracture or dislocation despite pre-existing CSCS.

\section{Conflict of Interest}

No potential conflict of interest relevant to this article was reported.

\section{References}

1. Harris JH, Yeakley JW. Hyperextension-dislocation of the cervical spine: ligament injuries demonstrated by magnetic resonance imaging. J Bone Joint Surg Br 1992;74:567-70.

2. Hendey GW, Wolfson AB, Mower WR, Hoffman JR; National Emergency X-Radiography Utilization Study Group. Spinal cord injury without radiographic abnormality: results of the National Emergency $\mathrm{X}$-Radiography Utilization Study in blunt cervical trauma. J Trauma 2002;53:1-4.

3. Tewari MK, Gifti DS, Singh P, et al. Diagnosis and prognostication of adult spinal cord injury without radiographic abnormality using magnetic resonance imaging: analysis of 40 patients. Surg Neurol 2005;63: 204-9.

4. Kothari P, Freeman B, Grevitt M, Kerslake R. Injury to the spinal cord without radiological abnormality (SCIWORA) in adults. J Bone Joint Surg Br 2000; 82:1034-7.

5. Yucesoy K, Yuksel KZ. SCIWORA in MRI era. Clin Neurol Neurosurg 2008;110:429-33.

6. Machino M, Yukawa Y, Ito K, et al. Can magnetic resonance imaging reflect the prognosis in patients of cervical spinal cord injury without radiographic abnormality? Spine (Phila Pa 1976) 2011;36:E156872.

7. Koyanagi I, Iwasaki Y, Hida K, Akino M, Imamura H, Abe H. Acute cervical cord injury without fracture or dislocation of the spinal column. J Neurosurg 2000; 93(1 Suppl):15-20.

8. Tator $\mathrm{CH}$. Clinical manifestations of acute spinal cord injury. In: Benzel EC, Tator CH; Aans Publications Committee, editors. Contemporary management of spinal cord injury. Park Ridge: American Association of Neurological Surgeons; 1995. p.15-26.
9. Saruhashi Y, Hukuda S, Katsuura A, Asajima S, Omura K. Clinical outcomes of cervical spinal cord injuries without radiographic evidence of trauma. Spinal Cord 1998;36:567-73.

10. Hardy AG. Cervical spinal cord injury without bony injury. Paraplegia 1977;14:296-305.

11. Shimada K, Tokioka T. Sequential MRI studies in patients with cervical cord injury but without bony injury. Paraplegia 1995;33:573-8.

12. Harrop JS, Sharan A, Ratliff J. Central cord injury: pathophysiology, management, and outcomes. Spine J 2006;6(6 Suppl):198S-206S.

13. Takahashi M, Harada Y, Inoue H, Shimada K. Traumatic cervical cord injury at C3-4 without radiographic abnormalities: correlation of magnetic resonance findings with clinical features and outcome. J Orthop Surg (Hong Kong) 2002;10:129-35.

14. Regenbogen VS, Rogers LF, Atlas SW, Kim KS. Cervical spinal cord injuries in patients with cervical spondylosis. AJR Am J Roentgenol 1986;146:277-84.

15. White AA 3rd, Johnson RM, Panjabi MM, Southwick WO. Biomechanical analysis of clinical stability in the cervical spine. Clin Orthop Relat Res 1975;(109):8596.

16. Okada S, Maeda T, Ohkawa Y, et al. Does ossification of the posterior longitudinal ligament affect the neurological outcome after traumatic cervical cord injury? Spine (Phila Pa 1976) 2009;34:1148-52.

17. La Rosa G, Conti A, Cardali S, Cacciola F, Tomasello F. Does early decompression improve neurological outcome of spinal cord injured patients? Appraisal of the literature using a meta-analytical approach. Spinal Cord 2004;42:503-12.

18. Chen TY, Dickman CA, Eleraky M, Sonntag VK. The role of decompression for acute incomplete cervical spinal cord injury in cervical spondylosis. Spine (Phila Pa 1976) 1998;23:2398-403.

19. Yamazaki T, Yanaka K, Fujita K, Kamezaki T, Uemura $\mathrm{K}$, Nose T. Traumatic central cord syndrome: analysis of factors affecting the outcome. Surg Neurol 2005; 63:95-9.

20. Itoh Y, Mazaki T, Koshimune K, Morita T, Mizuno S. Randomized controlled study of treatment for acute cervical cord injury with spinal canal stenosis but without radiographic evidence of trauma (SCIWORET): operative or conservative treatment. J Spine Res 2011;2:965-7. 
21. Kawano O, Ueta T, Shiba K, Iwamoto Y. Outcome of decompression surgery for cervical spinal cord injury without bone and disc injury in patients with spinal cord compression: a multicenter prospective study. Spinal Cord 2010;48:548-53.

22. Takao T, Morishita Y, Okada S, et al. Clinical relationship between cervical spinal canal stenosis and traumatic cervical spinal cord injury without major fracture or dislocation. Eur Spine J 2013;22:2228-31.

23. Morishita Y, Naito M, Wang JC. Cervical spinal canal stenosis: the differences between stenosis at the lower cervical and multiple segment levels. Int Orthop 2011;35:1517-22.
24. Morishita Y, Naito M, Hymanson H, Miyazaki M, Wu G, Wang JC. The relationship between the cervical spinal canal diameter and the pathological changes in the cervical spine. Eur Spine J 2009;18:877-83.

25. Katoh S, el Masry WS, Jaffray D, et al. Neurologic outcome in conservatively treated patients with incomplete closed traumatic cervical spinal cord injuries. Spine (Phila Pa 1976) 1996;21:2345-51.

26. Ishida Y, Tominaga T. Predictors of neurologic recovery in acute central cervical cord injury with only upper extremity impairment. Spine (Phila Pa 1976) 2002;27:1652-8. 